\title{
Rapid Detection of Aspergillus Fumigatus Growth Percentage by Microplate Reader
}

\author{
Lin Sun ${ }^{1}$, Jianye Shao ${ }^{1}$, XiaoLin Wang ${ }^{2}$, Bin Wang ${ }^{2}$, Wei Zhao ${ }^{2}$
}

\author{
${ }^{1}$ Department of Microbiology, College of Life Sciences, Qingdao University Shandong, China \\ ${ }^{2}$ Department of Basic Medical Sciences, Qingdao University, Shandong, China
}

\begin{abstract}
In this experiment, the OD value of different concentrations of Aspergillus fumigatus cultured for 48 hours was measured on a microplate reader using a 96-well plate. The absorbance-concentration curve was plotted according to the results, and the absorbance value was used instead of visual observation to quickly detect the growth of Aspergillus fumigatus. situation. The results showed that the OD value was between 0.05 and 0.06 when visually $50 \%$, and $100 \%$ when the OD value was greater than 0.17 . The use of the OD value can be a rapid method for observing the growth of Aspergillus fumigatus, which has the advantages of high efficiency, accuracy and high throughput compared with the visual method.
\end{abstract}

Keywords: Aspergillus fumigatus, Microplate Reader, OD, Value Growth Status

\section{Introduction}

Aspergillus fumigatus is an important conditional pathogenic fungus that causes invasive aspergillosis (IA) in immunocompromised hosts [1-2]. When patients with immunocompromised inhalation of Aspergillus spores, spores invade the lungs through the trachea, bronchi, and alveoli, causing initial invasive infections, often accompanied by dissemination of distant organs, in view of clinically not directly effective for Aspergillus fumigatus The method of determination, therefore, the study of the growth changes of Aspergillus fumigatus will become the first element of the treatment of Aspergillus fumigatus [3-4]. At present, the research of fungi directly uses visual inspection to directly observe its morphology. The method is simple and cannot be concluded quickly and effectively, and there is a certain error. In other literatures, since the number/concentration of the test bacteria is proportional to the optical density (OD) value of the bacterial suspension, the Aspergillus fumigatus suspension is measured by a spectrophotometer, but the method is also obvious. Disadvantages: the experimental operation is complicated, the cycle is long, and the bacteria are easily infected during repeated feeding. The fungus in the bacterial suspension will precipitate and the OD value will change, and the result is unstable [5-6]. The purpose of this paper is to measure the OD value of Aspergillus fumigatus in the state of bacterial suspension by using the microplate reader, draw the absorbance-concentration curve according to the OD value, and use a safe and reliable method to express the growth status of Aspergillus fumigatus quickly and accurately for later detection. The antibacterial ability of antimicrobial peptides provides a reliable method [7-8].

\section{MATERIALS AND METHODS \\ Strain activation}

The AMM medium suitable for the growth of Aspergillus fumigatus is configured as follows:

\begin{tabular}{ll}
\hline Reagent & dose \\
\hline glucose & $2 \mathrm{~g}$ \\
Salt solution & $4 \mathrm{ml}$ \\
Add water to & $200 \mathrm{ml}, 1 \mathrm{~N} \mathrm{NaoH}$ adjusts \\
ph to 6.5 & \\
Agar powder & $3 \mathrm{~g}(1.5 \%)$ \\
Autoclave $\left(121^{\circ} \mathrm{C}\right.$ & $20 \mathrm{~min})$ \\
Ammonium & $2 \mathrm{ml}$ \\
tartrate & $1 \mathrm{ml}$ \\
Supp solution & \\
\hline
\end{tabular}

Shake well, pour into the plate, and cool for use. WT was inoculated into AMM medium, incubated in a $37^{\circ} \mathrm{C}$ incubator for 72 hours, and spores were collected in an ultra-clean bench in an autoclaved EP tube for a concentration of $10^{7}$ cells $/ \mathrm{ml}$.

\footnotetext{
Visible absorption curve of bacterial suspension The prepared spores were dissolved in 1640 medium, and the concentration was adjusted to 105 cells $/ \mathrm{ml}$. The 1640 medium without the bacterial solution was used as a blank control, and the configured bacterial suspension was placed under a microplate reader to measure the OD value.
} 
Preparation of 96-well plate suspension and measurement of corresponding OD value

The freshly collected Aspergillus fumigatus spores were subjected to gradient dilution, and the concentration of each gradient was $1 \times 10^{2} 、 2 \times 10^{2}$ 、 $3 \times 10^{2} 、 4 \times 10^{2} 、 5 \times 10^{2} 、 6 \times 10^{2} 、 7 \times 10^{2} 、 8 \times 10^{2}, 9 \times 10^{2}$, $1 \times 10^{3}, 2 \times 10^{3}, 3 \times 10^{3}, 4 \times 10^{3}, 5 \times 10^{3}, 6 \times 10^{3}, 7 \times 10^{3} 、$ $8 \times 10^{3} 、 9 \times 10^{3}, 1 \times 10^{4} 、 2 \times 10^{4} 、 3 \times 10^{4} 、 4 \times 10^{4} 、 5 \times 10^{4}$ 、 $6 \times 10^{4} 、 7 \times 10^{4} 、 8 \times 10^{4} 、 9 \times 10^{4}$, and each concentration was used as a standard comparison (addition of the same medium and bacterial solution in the well), and the 1640 medium was used as a blank control. The 96-well plate was wrapped in aluminum foil and placed in an incubator at $37^{\circ} \mathrm{C}$ to prevent evaporation of water in the plate. The OD value of each well was measured after 48 hours of incubation.

\section{Data analysis}

All experiments were repeated three times. The results were expressed as mean \pm standard error (mean \pm SEM). Data were analyzed and plotted using GraphPad Prism5 software. $\mathrm{P}<0.05$ indicated that the difference was statistically significant.

\section{RESULTS}

\section{Measurement wavelength selection}

The reason why the spectrophotometer can measure the concentration of a liquid is because of the application of Lambert Beer's law, which describes the relationship between the intensity of light absorption at a certain wavelength and the concentration of the light absorbing material and the thickness of its liquid layer. Since the experiment uses a bacterial suspension, there is no strict limit on the wavelength for determining the optical density of the suspension. For convenience, we chose $620 \mathrm{~nm}$ as the measurement wavelength.

\section{Test plate observation}

From Figure 1, we can clearly see the change of turbidity of Aspergillus fumigatus wild type before and after culture. From A1 to A9, it is $1 \times 10^{2} 、 2 \times 10^{2}$ 、 $3 \times 10^{2} 、 4 \times 10^{2} 、 5 \times 10^{2} 、 6 \times 10^{2} 、 7 \times 10^{2} 、 8 \times 10^{2} 、 9 \times 10^{2}$, A11 is the standard control, A12 is the reference, only equal volume of 1640 medium is added, each concentration Repeat three times, followed by 103 and 104. It can be seen from Fig. 1A that all the wells before the culture are transparent liquids. After the culture, according to the B chart, the standard control wells (A11) are the most turbid, and the blank control wells (A12) are still transparent liquids. As the concentration of the bacteria increases, the turbidity of the medium is higher until the bottom of the hole is covered.

\section{Test OD value}

According to the microplate reader, the OD value of all the wells of the 96-well plate was measured, and the OD value-visual scatter plot was drawn according to the commonly used visual method, as shown in Fig. 2. As can be seen from the figure, the OD value of the well to which only the medium is added is about 0.05 , corresponding to Figure $3 \mathrm{~A}$. The OD value also increases as the concentration increases. When visually $50 \%$, the OD value is between 0.05 and 0.06 , corresponding to Figure $3 \mathrm{~B}$. When the $\mathrm{OD}$ value is greater than 0.17 , the visual inspection is $100 \%$, corresponding to Figure IIIC. D and $\mathrm{E}$ are the positive control and the negative control, respectively.

\section{Discussion}

Aspergillus fumigatus is an important pathogenic fungal pathogen of immunodeficient hosts. It is ubiquitous in people's periphery. Its spores are extremely small, floating in the air, and can enter the lungs with breathing. In the presence of moisture and sufficient nutrients, such as in mammalian lungs, dormant conidia disrupt metabolism and dormancy of the cell cycle. After a period of nuclear fission and isotropic growth (expansion), each conidia establishes a polar axis and then develops into invasive hyphae, causing invasive aspergillosis, which is also the current death of invasive aspergillosis. The highest rate [9-11].

At present, the clinical evaluation of the growth state of Aspergillus fumigatus is mostly by visual inspection, and the proportion of Aspergillus fumigatus in 96-well plates is directly observed [12-13]. The method is easy to understand and simple to operate, but there are also many problems. For example, the difference of the observers may result in different readings, resulting in a certain error range of the results. In addition, the visual methods are mostly read only $0 \%, 20 \%, 50 \%$ and $100 \%$, and can not accurately and effectively distinguish smaller intervals. The existence of these shortcomings also makes the visual method only a basic method auxiliary use [14-15]. In this experiment, the OD value is read by the microplate reader, and the percentage read by the visual method is replaced by the $\mathrm{OD}$ value, so that the whole experimental process is simpler in operation, the result is more accurate, and the human error is reduced as small as possible. Exist, and the value is more visualized. At the same time, since the culture identification in this experiment is carried out in a 96-well plate, it is not necessary to remove the culture solution and is not easily contaminated by bacteria, and is less interfered by environmental factors. Compared with the visual method, the microplate reader can simultaneously detect the OD value of 96 samples in a short time, which has the advantages of fast, accurate and high throughput [16-18].

There are also corresponding deficiencies in this experiment. The various experimental data obtained by using the microplate reader to read the OD value are limited to the liquid medium 1640. If other medium is used, the OD value should be re-measured and determined. However, the results of this 
experiment show that it is feasible to use the microplate reader to read the OD value of the 96-well plate instead of the visual method, which provides a new method for the next step to identify the antibacterial effect of the antibacterial substance.

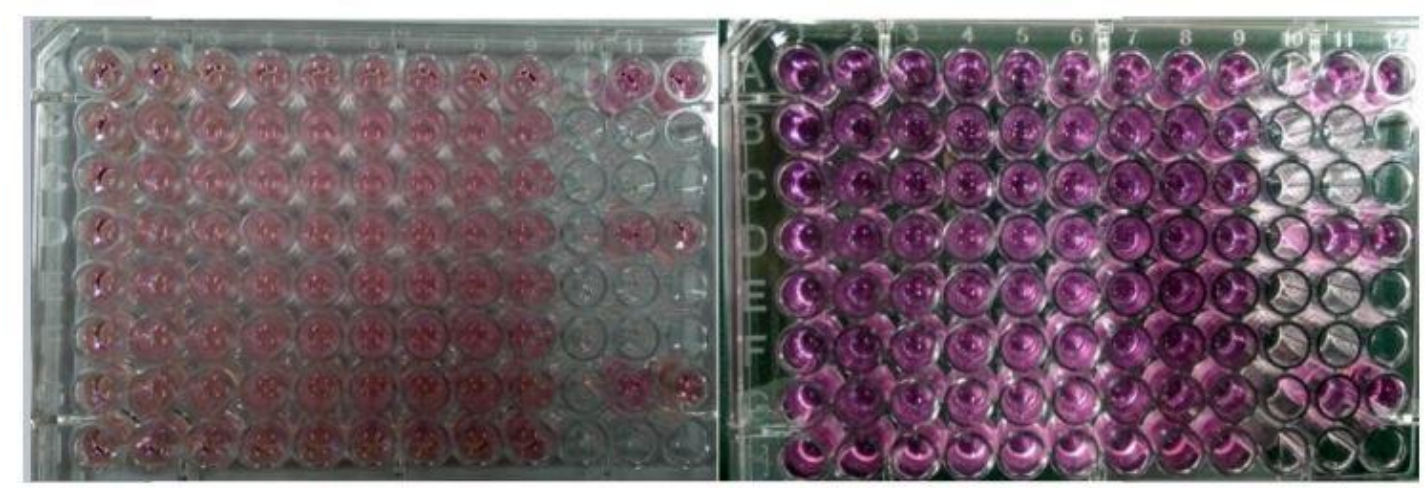

a

b

a:Growth of Aspergillus fumigatus in 96-well plates before culture; b:Growth of Aspergillus fumigatus in 96-well plate after culture.

Figure 1: Comparison of the growth status of Aspergillus fumigatus before and after 48 hours of culture.

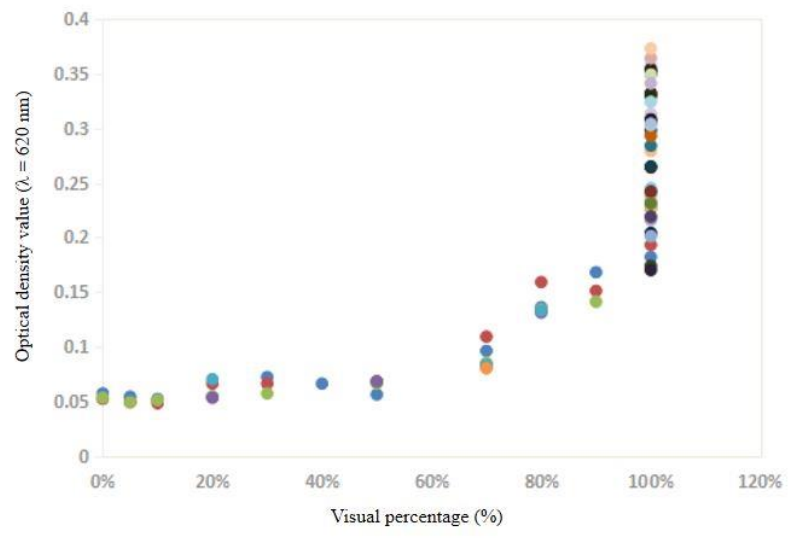

Figure 2: Correlation between visual and OD values after 48 hours of culture with Aspergillus fumigatus at different concentrations.

A

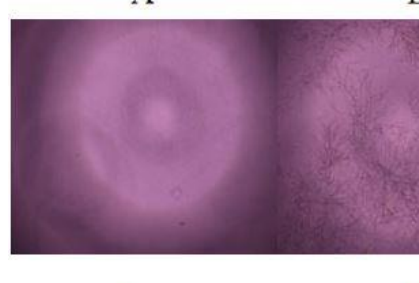

0
B

C

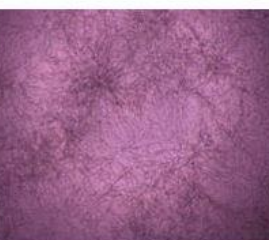

100
$\mathrm{D}$

E

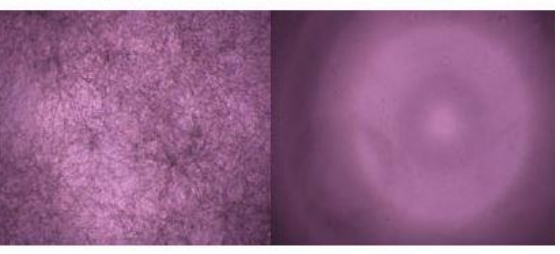

Standard control

Blank control

Figure 3: Observation of colony growth under an inverted microscope. A: Microscopic image when the visual value is $0 \% \mathrm{~B}$ : Microscopic image when the visual value is $50 \% 0 \mathrm{C}$ : Microscopic image when the visual value is $100 \% \mathrm{D}$ : Microscopic image of the standard control well E: blank control Microscopic image of the hole.

\section{References}

1. Xu Yuan,Chen Min,Liao Wanqing.The epidemiological status of invasive aspergillosis in China[J].Chinese Journal of Mycology,2018,13(01):57-60.

2. Zheng Shuai, Dai Jianghong. Pathogenicity and virulence factors of Aspergillus fumigatus[J]. Journal of Wuhan University of Light Industry, 2018, 37(02): 25-29+47.

3. Zhang Ming,Sun Wenbiao,Shi Yi.The resistance of Aspergillus fumigatus to azole antifungal drugs[J].Chinese Journal of Infection and Chemotherapy,2013,13(02):155-159.

4. Shao Jianye. Effect of PKA regulatory subunit on autophagy 
of Aspergillus fumigatus [D]. Qingdao University, 2018.

5. Gong Hongbo.Comparison of dual-wavelength and single-wavelength colorimetric determination of HBsAg by microplate reader[J].Chinese Community Physicians,2015,31(03):115+117.

6. Shi Yaoqiang, Zhang Yiyi, Liu Shuangyue. Research progress in the application of microplate reader detection technology [J]. Science and Technology Outlook, 2016, 26 (24): 73+107.

7. Zheng Lanyu. Use and Precautions of Microplate Reader [J]. Chinese Animal Husbandry and Veterinary Abstracts, 2018, 34 (03): 74.

8. $\mathrm{Hu}$ Wenbo. Feasibility Study on Determination of Alanine Aminotransferase by Microplate Reader [J]. Chinese Prescription Drugs, 2018, 16 (09): 38-39.

9. Shao Jianye, Sun Lin, Zhao Wei, Wang Bin.Expression of autophagy-related genes in autophagy of Aspergillus fumigatus [J].Biochemical,2018,4(03):116-118+121.

10. Zhang Wei, Wu Cuijiao, Zhao Wei, Shao Jianye, Wang Weiwei, Niu Qianqian, Wang Bin.Effects of PKA regulatory subunits on the germination of Aspergillus fumigatus by $\mathrm{MB}$ staining[J].Journal of Qingdao University(Natural Science Edition),2017,30( 02): 49-52.

11. WANG Weiwei,WU Cuijiao,ZHAO Wei,ZHANG Wei,WANG Bin.Effects of metal reductase FreB2 on iron absorption and oxidative stress response of Aspergillus fumigatus[J].Journal of Microbiology,2016,36(02):8-13.

12. Wang Min,Chai Tao,Lin Fancong,Wu Chunyan.Determination of White Rot Fungus Concentration in Suspension Culture System[J].Modern Chemical Industry,2010,30(09):90-92.

13. Zhang Lili, Wang Qiangxiong, Shen Liangliang, Yan Yanfen, Deng Shuwen, Zhao Jingjun. Molecular identification of clinical isolates of Aspergillus and sensitivity analysis of antifungal drugs in vitro[J].Journal of Tongji University(Medical Sciences),2018,39(01) :70-75.

14. Yang Qingwen, Guo Yidan, Zhou Huixin, Li Xiaoli, Li Wei.Experimental study on growth of agar bacteria with different concentrations of TTC plate count[J].Chinese Journal of Health Laboratory Technology,2012,22(01):62-63.

15. Determination of minimum inhibitory concentrations (MICs) of antibacterial agents by agar dilution $[\mathrm{J}]$. Clinical Microbiology and Infection,2000,6(9).

16. Yu Fei, Qin Yanfei, Wang Zhou, Sun Junfeng, Zhang Min, Xue Zhenglian. Determination of Lincomycin in Microplate Fermentation Broth by Microplate Reader [J/OL]. Chinese Journal of Antibiotics: 1-5[2018-1213].https://doi.org/10.13461/j.cnki.cja.006213.

17. Zheng Lanyu. Use and Precautions of Microplate Reader [J]. Chinese Animal Husbandry and Veterinary Digest, 2018, 34 (03): 74 .

18. Fang Fang, Liu Chunyan, Chen Guanshan, Yuan Pingchuan, Wang Hao. Stability of Exopolysaccharide from Alternaria alternata and Spectrophotometry to the Stability of Enzyme-labeled Method[J].Journal of Heilongjiang August First Land Reclamation University, 2017 , 29 (04): 72-77. 